\title{
Accuracy of Circuit Analysis Method using Wavelet Transform with Adaptive Resolutions
}

\author{
Masanori Oishi and Seiichiro Moro \\ Department of Electrical and Electronics Engineering \\ University of Fukui \\ Fukui 910-8507, Japan \\ Email: oishi@ppc8100.fuee.u-fukui.ac.jp, moro@u-fukui.ac.jp
}

\author{
Tadashi Matsumoto \\ Department of Electrical and Electronics Engineering \\ Fukui University of Technology \\ Fukui, 910-8505, Japan \\ Email: matsu@fukui-ut.ac.jp
}

\begin{abstract}
Recently, much attention have been paid to the methods for circuit analysis using wavelet transform. In particular, we have proposed the method which can choose the resolution of the wavelet adaptively. This method can fully bring out the orthogonal and the multiresolution properties of the wavelet, and the efficiency of the calculation can be improved. In this paper, we evaluate the accuracy of the adaptive resolution method to analyze the circuit using wavelet transform.
\end{abstract}

\section{INTRODUCTION}

The wavelet transform has been often used in signal processing because of its orthogonality and multiresolution property. Recently, much attention has been paid to the method for circuit analysis using wavelet transform [7]-[14]. In particular, Barmada et al. have proposed the Fourier-like approach for the circuit analysis using the wavelet transform [11]. In this method, the integral and differential operator matrices are introduced to the analysis, and the differential and integral equations are transformed into the algebraic equations like as using Fourier or Laplace transforms. Moreover, the method can treat time varying and nonlinear circuits. Therefore, this method is useful for various circuit analyses.

However, in that method, the use of Daubechies wavelet makes the handling of the operator matrices complicated, especially, in the edges of the interval. Thus, we have proposed the circuit analysis method using Haar wavelet transform [14]. The Haar wavelet is easy to handle itself, and the operator matrices using the Haar wavelets are easily derived by introducing the block pulse functions [5], [6]. Moreover, the proposed method can treat the nonlinear time varying circuits.

In addition, Haar wavelets have the merit to be able to analyze the trajectory near the singular points where the trajectory moves rapidly with high resolution because of the orthogonality and localization property of the wavelet functions. As circuit analysis methods using this merit, some methods were proposed to pick out the ranges automatically where the trajectory moves rapidly near singular points. Thus we have proposed the method for circuit analysis using wavelet transform with adaptive resolutions [16]. In this method, the result of the multiresolution analysis is used to choose the range to be analyzed more precisely. It makes the adaptive choice of the wavelet resolution possible, and as a result, the efficient calculation can be achieved. However, the accuracy, which is the other important aspect of numerical calculation, has not been discussed well until now.

In this paper, we evaluate the accuracy of the adaptive resolution method using the stiff ordinary differential equations. From the results, we can show that the accuracy can be drastically improved by adding the small number of plots. This property will be dominantly seen in the stiff system and we expect the method can be the powerful tool to analyze the power electronic circuits and the hybrid dynamical systems.

\section{HAAR WAVELET MATRIX}

Haar functions are defined on interval $[0,1)$ as follows,

$$
\begin{gathered}
h_{0}=\frac{1}{\sqrt{m}}, \\
h_{i}=\frac{1}{\sqrt{m}} \times \begin{cases}2^{\frac{j}{2}}, & \frac{k-1}{2^{j}} \leq t<\frac{k-\frac{1}{2}}{2^{j}}, \\
-2^{\frac{j}{2}}, & \frac{k-\frac{1}{2}}{2^{j}} \leq t<\frac{k}{2^{j}}, \\
0, & \text { otherwise in }[0,1),\end{cases} \\
i=0,1, \cdots, m-1, m=2^{\alpha},
\end{gathered}
$$

where $\alpha$ is positive integer, and $j$ and $k$ are nonnegative integers which satisfy $i=2^{j}+k$, i.e., $k=0,1, \cdots, 2^{j}-1$ for $j=0,1,2, \cdots$.

$\vec{y}$ is $m \times 1$-dimensional vector whose elements are the discretized expression of $y(t)$ and $\vec{c}$ is $m \times 1$-dimensional coefficient vector. $H$ is $m \times m$-dimensional Haar wavelet matrix defined as

$$
H=\left[\begin{array}{c}
\vec{h}_{0} \\
\vec{h}_{1} \\
\vdots \\
\vec{h}_{m-1}
\end{array}\right],
$$

where $\vec{h}_{i}$ is $1 \times m$-dimensional Haar wavelet basis vector whose elements are the discretized expression of $h_{i}(t)$. Using these vectors and matrix, Haar wavelet transform and inverse Haar wavelet transform are described as follows, respectively,

$$
\begin{gathered}
\vec{c}=H \vec{y}, \\
\vec{y}=H^{T} \vec{c}\left(=H^{-1} \vec{c}\right) .
\end{gathered}
$$




\section{INTEgRAL AND DifFERENTIAL OPERATOR MATRICES USING HAAR WAVELET}

The basic idea of the operator matrix has been firstly introduced by using Walsh function [5]. However, in logical way, the matrices introduced by block pulse function are more fundamental [4], [5]. The block pulse function is the set of $m$ rectangular pulses which have $1 / m$ width and are shifted $1 / m$ each other.

The integral operator matrix of the block pulse function matrix $B$ is defined as the following equation [5], [6].

$$
\begin{gathered}
\int_{0}^{i} B(\tau) d \tau \equiv Q_{B} \cdot B(t), \\
Q_{B(m \times m)}=\frac{1}{m}\left[\frac{1}{2} I_{(m \times m)}+\sum_{i=1}^{\infty} P_{(m \times m)}^{i}\right],
\end{gathered}
$$

where $B(t)$ is $m \times m$-dimensional matrix whose elements are the discretized expression of the block pulse functions $b_{i}(t)$, $i=0,1, \cdots, m-1$ and

$$
P_{(m \times m)}^{i}=\left[\begin{array}{l|c}
0 & \mathrm{I}_{(m-i) \times(m-i)} \\
\hline 0_{(i \times i)} & 0
\end{array}\right]
$$

for $i<m$,

$$
P_{(m \times m)}^{i}=0_{(m \times m)}
$$

for $i \geq m$. And the inverse matrix $Q_{B(m \times m)}^{-1}$ is calculated as follows [5]:

$$
Q_{B(m \times m)}^{-1}=4 m\left[\frac{1}{2} I_{(m \times m)}+\sum_{i=1}^{m-1}(-1)^{i} P_{(m \times m)}^{i}\right] .
$$

As the Haar wavelet matrix $H$ is the set of the orthogonal functions, the integral matrix of $H$ is given as follows:

$$
Q_{H}=H Q_{B}^{T} H^{-1}=H Q_{B}^{T} H^{T} .
$$

Similarly, the differential matrix of $H$ can be written as

$$
Q_{H}^{-1}=H\left(Q_{B}^{T}\right)^{-1} H^{-1}=H\left(Q_{B}^{T}\right)^{-1} H^{T} .
$$

IV. HAar WaVelet Expression OF BRANCH CHARACTERISTICS OF NONLINEAR TIME VARYING

\section{CiRCUIT ELEMENTS}

As the Haar wavelet is defined on interval $[0,1)$, the generic interval $\left[t_{\min }, t_{\max }\right)$ can be rescaled by a new variable $\tau$ on $[0,1)$, where $\left[t_{\max }-t_{\min }\right) \tau+t_{\min }$. In this paper, $t_{\min }=0$ without loss of generality, then capacitance $c[\mathrm{~F}]$ and inductance $l$ [H] are scaled to $C=c / t_{\max }$ and $L=l / t_{\max }$, respectively.

Next, we show the Haar wavelet expression of branch characteristics of nonlinear time varying circuit elements for the expression in wavelet domain. See details in [14].

\section{Capacitor:}

$$
\begin{aligned}
v(t) & =v\left(0_{-}\right)+\frac{1}{C} \int_{0}^{t} i(\tau) d \tau, \quad v_{0}:=v\left(0_{-}\right), \\
V & =V_{0}+C_{w}^{-1} Q_{H} I \\
\text { or } \quad I & =C_{w} Q_{H}^{-1}\left[V-V_{0}\right],
\end{aligned}
$$

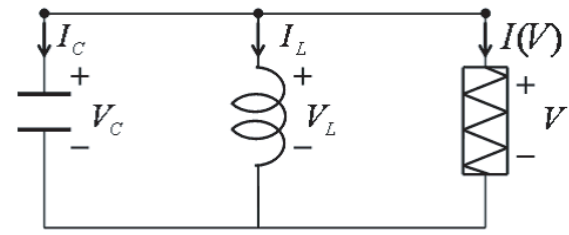

Fig. 1. Van der Pol oscillator circuit.

$$
C_{w}=H \operatorname{diag}\left[C\left(i_{0}, t_{0}\right), C\left(i_{1}, t_{1}\right), \cdots, C\left(i_{m-1}, t_{m-1}\right)\right] H^{T}
$$

Inductor:

$$
\begin{aligned}
i(t) & =i\left(0_{-}\right)+\frac{1}{L} \int_{0}^{t} v(\tau) d \tau, \quad i_{0}:=i\left(0_{-}\right), \\
I & =I_{0}+L_{w}^{-1} Q_{H} V \\
\text { or } \quad V & =Q_{H}^{-1} L_{w}\left[I-I_{0}\right] . \\
L_{w}=H \operatorname{diag} & {\left[L\left(i_{0}, t_{0}\right), L\left(i_{1}, t_{1}\right), \cdots, L\left(i_{m-1}, t_{m-1}\right)\right] H^{T} }
\end{aligned}
$$

$\underline{\text { Resistor: }}$

$$
\begin{aligned}
v(t) & =R i(t), \\
V & =R_{w} I, \quad R_{w}=\operatorname{diag}[R] .
\end{aligned}
$$

$$
R_{w}=H \operatorname{diag}\left[R\left(i_{0}, t_{0}\right), R\left(i_{1}, t_{1}\right), \cdots, R\left(i_{m-1}, t_{m-1}\right)\right] H^{T}
$$

\section{Circuit Analysis using Adaptive Resolution With MultiResolution ANALYsis [16]}

In this section, we show a previous method for automatically picking out the ranges to use the adaptive resolution by analyzing a example as follow.

The circuit model is a van der Pol oscillator circuit as shown in Fig. 1. The characteristic of nonlinear resistor of this circuit is;

$$
i(v)=-g_{1} v+g_{3} v^{3}=\left(-g_{1}+g_{3} v^{2}\right) v,
$$

and branch equations in the wavelet domain are shown as;

$$
\begin{aligned}
& I_{C}=C_{\omega} Q_{H}^{-1}\left(V-V_{0}\right), \\
& I_{L}=I_{L 0}+L_{\omega}^{-1} Q_{H} V, \\
& L_{w}=H \operatorname{diag}\left[L\left(i_{0}, t_{0}\right), L\left(i_{1}, t_{1}\right), \cdots, L\left(i_{m-1}, t_{m-1}\right)\right] H^{T}, \\
& I(V)=G_{w} V .
\end{aligned}
$$

where $H \vec{v}\left(0_{-}\right)=V_{0}, H \vec{i}_{L}\left(0_{-}\right)=I_{L 0}, H \vec{v}=V$, and $G_{w}=$ $H \operatorname{diag}\left[-g_{1}+g_{3} v_{0}^{2},-g_{1}+g_{3} v_{1}^{2}, \cdots,-g_{1}+g_{3} v_{m-1}^{2}\right] H^{T}$. In this example, we take $g_{1}=2, g_{3}=1, C=0.05[\mathrm{~F}], L=0.05[\mathrm{H}]$, $v_{C}\left(0_{-}\right)=0[\mathrm{~V}]$, and $i_{L}\left(0_{-}\right)=1[\mathrm{~A}]$.

From these characteristics, the nonlinear algebraic equations are solved numerically with respect to $V$, and calculation result for $m=64$ without the adaptive resolution is shown in Fig. 2. If the waveforms have the singular points, more detailed analyses are needed by using the smaller intervals. However, it makes the calculation cost higher, for example, by taking $m=128$. Even if waveforms are predicted by experimentally imagine, we cannot predict the singular points for every circuit 


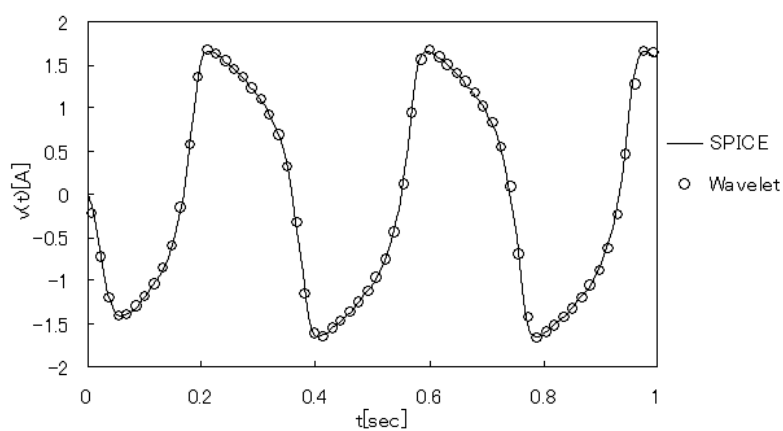

Fig. 2. Calculation result for $m=64$ without adaptive resolution [16].

$f(6)$
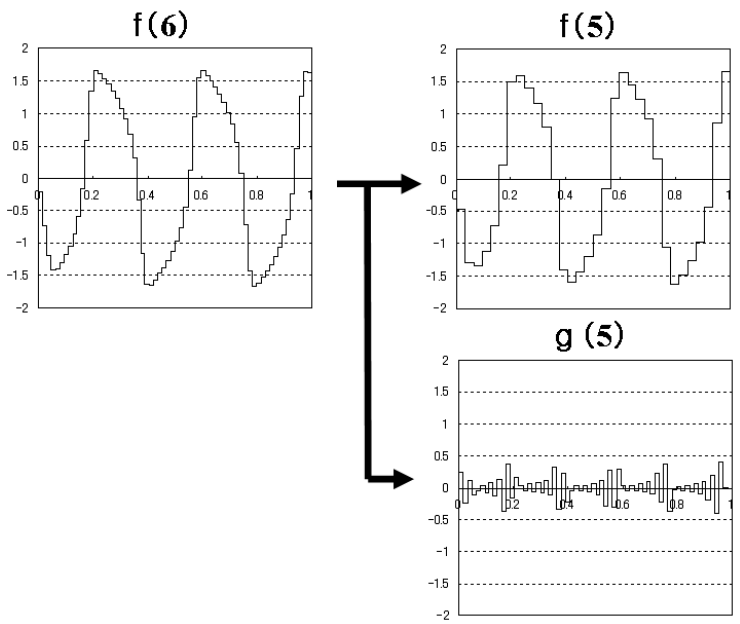

Fig. 3. Multiresolution analysis for $m=64$.

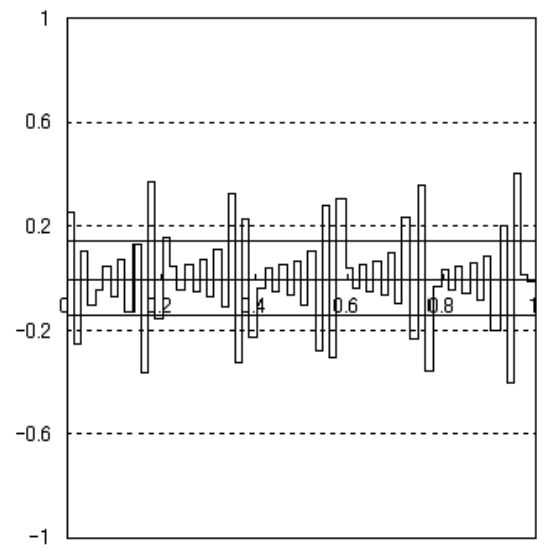

Fig. 4. Multiresolution analysis $g(5)$.

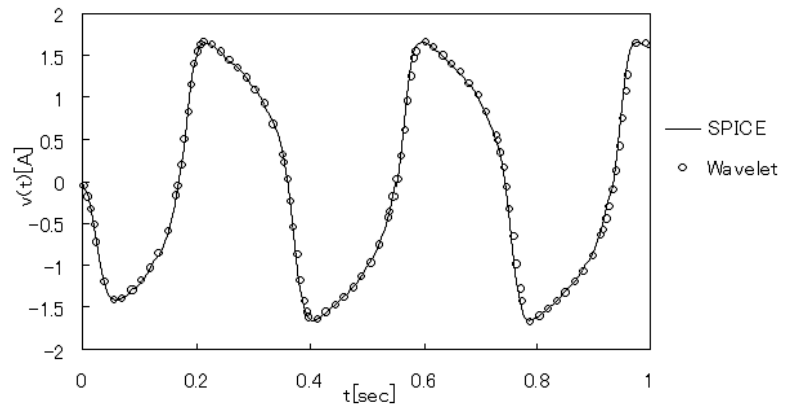

Fig. 5. Calculation result with adaptive resolution.

TABLE I

Picked Out By MultiResolution.

\begin{tabular}{ccc}
\hline picked out range & total plots $p$ & sampling width \\
\hline $0-0.02$ & 2 & $1 / 4$ \\
$0.16-0.21$ & 4 & $1 / 8$ \\
$0.35-0.40$ & 4 & $1 / 8$ \\
$0.54-0.59$ & 4 & $1 / 8$ \\
$0.73-0.77$ & 4 & $1 / 8$ \\
$0.91-0.96$ & 4 & $1 / 8$ \\
\hline
\end{tabular}

in the real world. To overcome this problem, we proposed the method using the results of the multiresolution analysis [16].

Figure 3 shows the results of the multiresolution analysis of the waveform shown in Fig. 2. $f(j)$ indicates the amounts of the results for $0,1, \cdots, j-1$-th resolution wavelets and $g(j)$ indicates the $j$-th resolution ones. Note that $f(6)=f(5)+$ $g(5)$. Because $g(j)$ can be considered as the error between the lower and the higher resolution cases, if $g(j)$ is large then the approximation is not sufficient. On the other hand, if $g(j)$ is small, it is considered that the approximation has already achieved. In this method, we take the threshold $\varepsilon$ that mean value of absolute value for $g(j)$, then pick out the ranges for $g(j)>|\varepsilon|$, and the thin lines indicate the threshold $\pm \varepsilon$ shown in Fig. 4. In those ranges, we analyze the circuit again with higher resolution wavelets. To avoid the over sampling, we set the sampling width as $1 / 2^{\alpha}$ to satisfy $2^{\alpha-1}<2 p \leq 2^{\alpha}$ where $p$ is the number of plots in the picked out ranges. Table I shows the relationship between the picked out ranges and the sampling width. The result for this method is shown in Fig. 5. The total number of plots is 97 and it is less than 128 that are the number of plots for the case of one higher resolution analysis.

From these results, it is confirmed that the method has ability to solve numerically the differential equations in efficient way. On the other hand, the accuracy of the numerical calculation methods is also quite important. In the next section, we discuss the accuracy of the method using the simple stiff linear ordinary differential equations. 


\section{ERror Evaluation USING STIFF Linear DIFFERENTIAL EQUATIONS}

In this section, we show the adaptive resolution method using a simple example. We apply the proposed method to the linear time invariant system;

$$
\begin{aligned}
& \dot{x}^{(1)}(t)=81 x^{(1)}+216 x^{(2)}+45 \cos 9 t-3 \sin 9 t, \\
& \dot{x}^{(2)}(t)=-216 x^{(1)}-459 x^{(2)}-81 \cos 9 t+3 \sin 9 t,
\end{aligned}
$$

and with the initial conditions;

$$
x_{0}^{(1)}=x^{(1)}(0)=\frac{4}{3}, x_{0}^{(2)}=x^{(2)}(0)=\frac{2}{3} .
$$

From these ordinary differential equations, we have the following exact solutions;

$$
\begin{aligned}
& x^{(1)}(t)=2 e^{-27 t}-e^{-351 t}+\frac{1}{3} \sin 9 t, t \geq 0, \\
& x^{(2)}(t)=e^{-27 t}-2 e^{-351 t}+\frac{1}{3} \sin 9 t, t \geq 0,
\end{aligned}
$$

Because the difference between two time constants is large, the system is stiff ordinary differential equations. Although this example does not treat actual circuit, it is not meaningless because the state equations of the electric circuits are usually described by ordinary differential equations.

In this case, we have the following Haar wavelet expression of Eq. (16);

$$
\begin{array}{r}
{\left[\begin{array}{c|c}
Q_{H(m \times m)}^{-1} & 0 \\
\hline 0 & Q_{H(m \times m)}^{-1}
\end{array}\right] \cdot\left[\begin{array}{c}
X_{m}^{(1)} \\
X_{m}^{(2)}
\end{array}\right]=} \\
{\left[\begin{array}{c|c}
81\left(\mathrm{I}_{(m \times m)}\right) & 216\left(\mathrm{I}_{(m \times m)}\right) \\
\hline-216\left(\mathrm{I}_{(m \times m)}\right) & -459\left(\mathrm{I}_{(m \times m)}\right)
\end{array}\right]} \\
\cdot\left[\begin{array}{c}
X_{m}^{(1)} \\
X_{m}^{(2)}
\end{array}\right] \\
+\left[\begin{array}{c}
W_{m}^{(1)} \\
W_{m}^{(2)}
\end{array}\right],
\end{array}
$$

where the transform of the input parts of sine wave and cosine wave are described by $W_{m}^{(1)}$ and $W_{m}^{(2)}$.

Then the method is possible to analyze the differential equations algebraically, and we show the results for $m=64$ and 128 without the adaptive resolution in Figs. 6 and 7, respectively. The approximation in the range of early part is not sufficient for $m=64$ case, while the range of late part is achieved. On the other hand, for $m=128$ case, better approximation is achieved even in the range of early part, but the size of the matrix of Eq. (18) becomes much larger. Therefore, it is necessary to analyze the trajectory which moves rapidly in early part more precisely while the analysis of the other part should be remained in lower resolution. Applying the method shown in [16] to this example, we take the threshold as the mean value of absolute values of multiresolution analysis as shown in Fig. 8. Only in the range where the traces of Fig. 8 exceed the threshold are analyzed by higher resolution wavelets.

The result with the adaptive resolution for the proposed method is shown in Fig. 9. The total number of plots is 87 and it is much less than 128 that are the number of plots for the case of one higher resolution analysis. Therefore, the

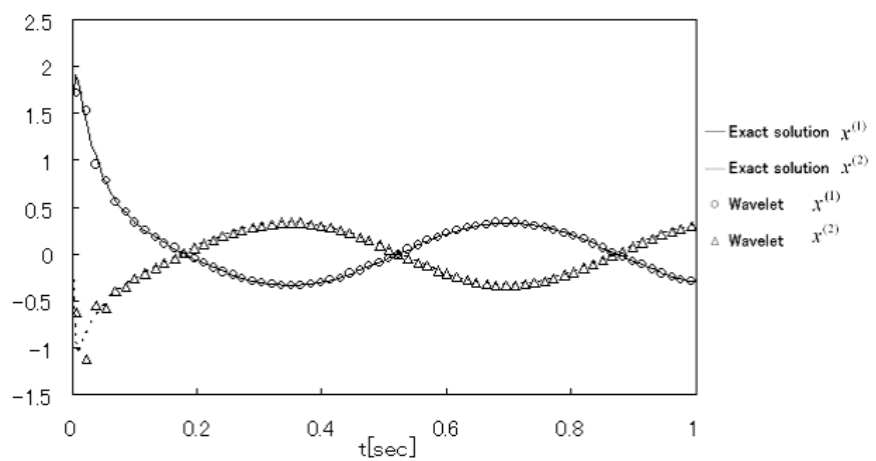

Fig. 6. Calculation result for $m=64$ without adaptive resolution [16].

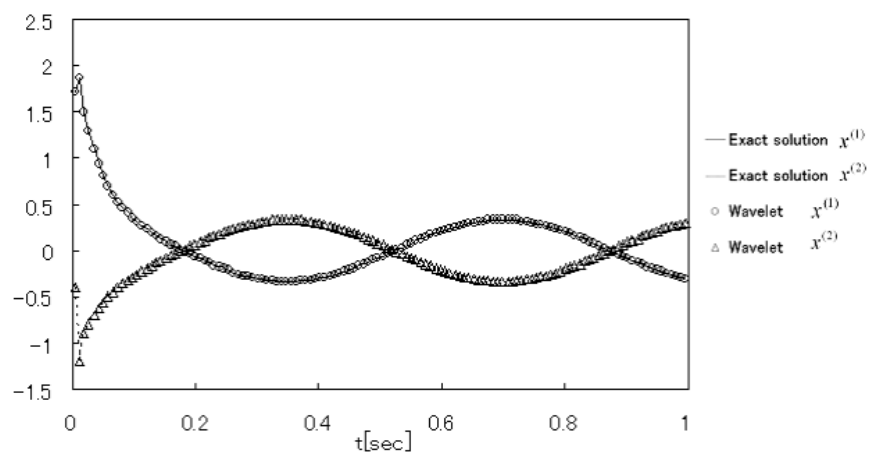

Fig. 7. Calculation result for $m=128$ without adaptive resolution [16].

more efficient approximation can be achieved when we use the method using wavelet transform with adaptive resolution shown in [16]. However, the accuracy of this method has not been discussed until now. Next, we consider the accuracy of the method by calculating the error between the exact solutions and the calculated results.

To evaluate the accuracy of the method, we introduce two types of errors, the mean relative error (MRE) and mean absolute error (MAE) defined as

$$
\begin{aligned}
& \text { MRE }=\frac{1}{N} \sum_{i=1}^{N}\left|\frac{\hat{x}_{j}^{(i)}-x_{j}^{(i)}}{x_{j}^{(i)}}\right| \\
& \text { MAE }=\frac{1}{N} \sum_{i=1}^{N}\left|\hat{x}_{j}^{(i)}-x_{j}^{(i)}\right|
\end{aligned}
$$
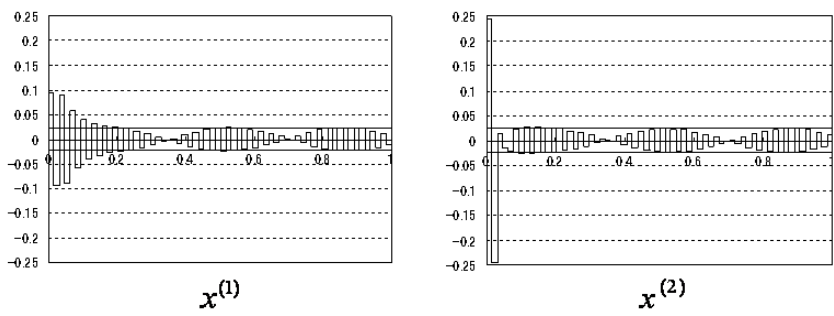

Fig. 8. Multiresolution analysis for $m=64(\mathrm{~g}(5))$. 


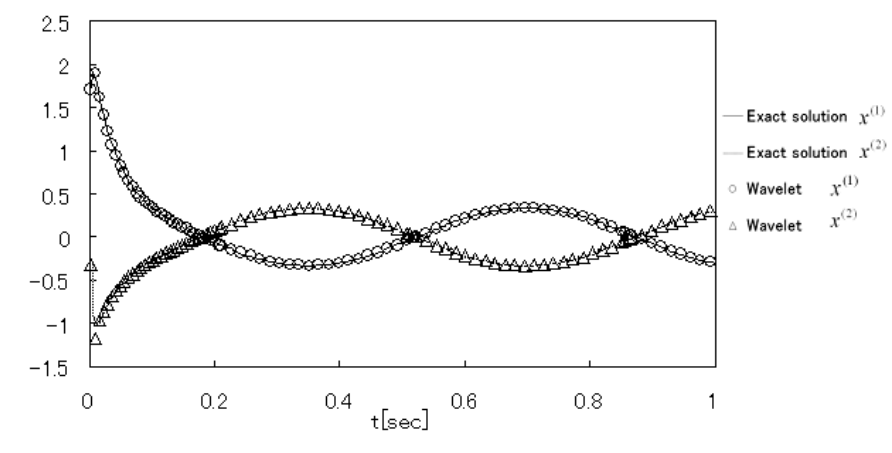

Fig. 9. Calculation result with adaptive resolution.

TABLE II

MRE AND MAE FOR THE METHODS WITH AND WITHOUT ADAPTIVE RESOLUTIONS.

\begin{tabular}{c|c|c|c}
\hline$x_{1}$ & $N=64$ & $N=87$ & $N=128$ \\
\hline MRE & 0.064822 & 0.005437 & 0.001861 \\
\hline MAE & 0.006615 & 0.002676 & 0.002094 \\
\hline \multicolumn{4}{|c}{} \\
\hline$x_{2}$ & $N=64$ & $N=87$ & $N=128$ \\
\hline MRE & 0.020335 & 0.009233 & 0.005499 \\
\hline MAE & 0.013499 & 0.005049 & 0.004107 \\
\hline
\end{tabular}

where $j=1,2$ and $x_{j}^{(i)}$ is $i$-th plot of the exact solution $x_{j}$ and $\hat{x}_{j}^{(i)}$ is $i$-th plot of the approximated solution of $x_{j} . N$ is the total number of plots.

From the results shown in this section, the errors of $x_{1}$ and $x_{2}$ of Eq. (17) and (18) are calculated as shown in Table II. In this table, the results for $N=64$ and $N=128$ correspond to the case without adaptive resolution (Fig. 6 and 7, respectively) and the results for $N=87$ corresponds to the case with adaptive resolution (Fig. 9). From these results, the accuracy is drastically improved when we use the adaptive resolution method comparing with $N=64$ case. Though it is slightly worse than the case of $N=128$, the number of the plots is much less and the calculation costs can be reduced well. This property will be seen dominantly in the stiff system such as the example shown above. This means the adaptive resolution method is highly suitable for analyses of the power electronics circuits and the hybrid dynamical systems, which include the drastic switching of the dynamics. Therefore this method can be powerful tool to analyze such systems.

\section{CONCLUSION}

In this paper, we have evaluated the accuracy of the circuit analysis method using wavelet transform with adaptive resolutions. By calculating both MRE and MAE, the accuracy improves drastically by adding the small number of plots by adaptive resolution method. The efficiency of this method has been already shown in [16] and the accuracy improvement is also shown in this paper. It is considered that such properties can be dominantly seen in the stiff systems because the good approximation has been already achieved in the most of the interval. This means the adaptive resolution method is highly suitable for analyses of the power electronics circuits and the hybrid dynamical systems, which include the drastic switching of the dynamics. Therefore this method can be powerful tool to analyze such systems. The realization of such tools is our future problem.

\section{REFERENCES}

[1] A. Haar, "Zur Theorie der orthogonalem funktionen System," Math. Ann., vol.69, pp.331-371, 1910.

[2] G. Strang, "Wavelet transforms versus Fourier Transforms," Bull. Amer. Math. Soc., vol.28, no.2, pp.288-305, Apr. 1993.

[3] C.F. Chen and C.H. Hsiao, "Haar wavelet method for solving lumped and distributed-parameter systems," IEE Proc. of Control Theory Appl., vol.144, no.1, pp.87-93, Jan. 1997.

[4] J.L. Wu, C.H. Chen, and C.F. Chen, "A model reduction via Haar wavelet," Int. J Control and Intelligent Systems, vol.29, no.2, pp.29-32, 2001.

[5] C.F. Chen, Y.T. Tsay, and T.T. Wu, "Walsh operational matrices for fractional calculus and their application to distributed systems," J. Franklin Institute, vol.303, no.3, pp.267-284, Mar. 1977.

[6] J.L. Wu, C.H. Chen, and C.F. Chen, "Numerical inversion of Laplace transform using Haar wavelet operational matrices," IEEE Trans. Circuits Syst. I, vol.48, no.1, pp.120-122, Jan. 2001.

[7] D. Zhou, X. Li, W. Zhang and W. Cai, "Nonlinear circuit simulation based on adaptive wavelet method," Proc. of IEEE International Symposium on Circuits and Systems (ISCAS'97), pp.1720-1723, June 1997.

[8] T. Matsumoto, T. Araki, and S. Moro, "Solving numerically stiff differential equations based on adaptive wavelet collocation methods," Proc. of IEEE International Symposium on Intelligent Signal Processing and Communication Systems (ISPACS'99), pp.669-672, Dec. 1999.

[9] D. Zhou and W. Cai, "A fast wavelet collocation method for high-speed circuit simulation," IEEE Trans. Circuits Syst. I, vol.46, no.8, pp.920-930, Aug. 1999.

[10] D. Zhou, W. Cai, and W. Zhang, "An adaptive wavelet method for nonlinear circuit simulation," IEEE Trans. Circuits Syst. I, vol.46, no.8, pp.931-938, Aug. 1999.

[11] S. Barmada and M. Raugi, "A general tool for circuit analysis based on wavelet transform," Int. J. Circuit Theory Appl., vol.28, no.5, pp.461-480, 2000.

[12] X. Li, B. Hu, X. Ling, and X. Zeng, "A wavelet-balance approach for steady-state analysis of nonlinear circuits," IEEE Trans. Circuits Syst. I, vol.49, no.5, pp.689-694, May 2002.

[13] G. Antonini and A. Orlandi, "A wavelet-based timedomain solution for PEEC circuit," IEEE Trans. Circuits Syst. I, vol.47, no.11, pp.1634-1639, Nov. 2000.

[14] A. Ohkubo, S. Moro, and T. Matsumoto, "A method for circuit analysis using Haar wavelet transforms," Proc. of IEEE Midwest Symposium on Circuits and Systems (MWSCAS'04), vol.3, pp.399-402, July 2004.

[15] Y. Nievergelt, Wavelets Made Easy, Birkhauser, 1999.

[16] M. Oishi, S. Moro, and T. Matsumoto, "A Method for Circuit Analysis using Haar Wavelet Transform with Adaptive Resolution," Proc. of International Symposium on Nonlinear Theory and its Applications (NOLTA'08), pp.369-372, Sep. 2008. 